\title{
会i- \\ Características cognitivas del emprendedor social en España
}

\author{
Catalina Nicolás Martínez \\ Universidad de Murcia, Facultad de Economía y Empresa. \\ catalina.nicolas@um.es \\ Alicia Rubio Bañón \\ Universidad de Murcia, Facultad de Economía y Empresa.
}

Resumen: Los emprendedores sociales son personas que, observando problemas sociales generalmente desatendidos por gobiernos e instituciones públicas, deciden resolver estas situaciones creando nuevas empresas. Son escasos los estudios empíricos que profundizan en el conocimiento de este grupo de emprendedores y, en particular, en las variables que inciden en la decisión de crear una empresa social. Esta investigación, utilizando los datos del proyecto Global Entrepreneurship Monitor (GEM), analiza la influencia en la decisión de convertirse en emprendedor social de tres variables cognitivas, miedo al fracaso, percepción de oportunidades y autoeficacia. Además, se comparan los 
resultados obtenidos para los emprendedores sociales con una muestra de emprendedores comerciales. La principal conclusión de este trabajo es que la influencia de las variables cognitivas en el perfil de los emprendedores es diferente.

Palabras clave: Emprendimiento, emprendedores sociales, miedo al fracaso, autoeficacia, percepción de oportunidades.

\section{Cognitive characteristics of the social entrepreneur in Spain}

Abstract: Social entrepreneurs are people who know that there are several social problems forgotten by governments and public institutions and decide to solve these situations creating new business. Few empirical studies deepen the knowledge of this group of entrepreneurs, and particulary the literature focusing on the variables that affect the decision to create a social enterprise is scarce. This research, using Global Entrepreneurship Monitor (GEM) data, analyzes the influence on the decision of becoming a social entrepreneur of three cognitive variables, fear of failure, perception of opportunities and self-efficacy. Moreover, we compare the results obtained for social entrepreneurs with a sample of commercial entrepreneurs. The main conclusions of this paper is that the influence of cognitive variables on entrepreneurs profile is different.

Keywords: Entrepreneurship, social entrepreneurs, fear of failure, selfefficacy, perception of opportunities. 


\section{Características cognitivas del emprendedor social en España}

Recibido: $17 / 1 / 2018$

Aceptado: 23/5/2018

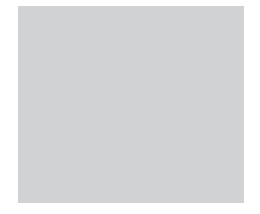

Catalina

Nicolás Martínez

Alicia

Rubio Bañón

\section{INTRODUCCIÓN}

Cuando un individuo decide iniciar una empresa, además de generar rentas de las que se beneficia, desarrolla una labor social en su entorno, contribuyendo indirectamente a la creación de empleo y riqueza. Existe un debate abierto en la literatura entre quienes argumentan que el emprendimiento comercial y social no deben distinguirse, ya que ambos tipos generan valor social (Acs et al., 2011), y los que sitúan el emprendimiento social como aquel con particularidades que le diferencian del comercial (Austin et al., 2006). La mayoría de investigaciones coinciden en que, aunque ambos emprendimientos puedan generar valor social, para el social crear este tipo de valor es su verdadera razón de ser (Dees, 1998; Seelos y Mair, 2007; Zahra et al., 2009; Meyskens et al., 2010; Kistruct y Beamish, 2010; Lepoutre et al., 2013; Nicolás et al., 2018).

El emprendimiento social no es un fenómeno reciente (Dees, 2001). Si se atiende a la historia es posible encontrar casos como la iniciativa Montes de Piedad, que data del siglo XV, la cual aplica soluciones prácticas a problemas sociales, combinando la innovación, la captación de fondos y la oportunidad (Muñoz Serrulla, 2004; Nicolás Martínez, 2014).

Aunque España presenta una baja tasa de actividad emprendedora social $^{1}$ (0,53 según el De la Vega et al., 2010), cada vez más personas inician empresas sociales en este país. Así lo

${ }^{1}$ Porcentaje de la población de 18-64 años involucrada en iniciativas emprendedoras sociales de entre 0 y 3,5 años. 
muestran asociaciones como Ashoka España, la cual ve incrementado el número de emprendedores sociales que la forman año tras año (Ashoka España, 2018).

Pese a ser un fenómeno empresarial que se está desarrollando desde hace siglos, el estudio sobre emprendimiento social aún es reciente (Short et al., 2009). En los 80, gracias a Bill Drayton, fundador de Ashoka, se acuña el término emprendedor social, pero no es hasta los 90 cuando se produce el verdadero avance científico con trabajos de Greg Dees (Bornstein y Davis, 2010).

Desde esta década, el emprendimiento social ha generado numerosos trabajos, la mayoría conceptuales y cualitativos (Lepoutre et al., 2013), que estudian y delimitan el concepto (Seelos y Mair, 2007), ponen de manifiesto sus principales características (Peredo y Chrisman, 2006), se centran en los aspectos éticos (Zahra et al., 2009), buscan marcos teóricos (Meyskens et al., 2010), analizan sus formas jurídicas (Kistruct y Beamish, 2010) o profundizan en las diferencias entre el emprendedor social y el comercial (Austin et al., 2006).

El objetivo de este trabajo es ampliar el conocimiento, que actualmente es muy escaso, de la influencia que en el individuo tienen, a la hora de convertirse en emprendedor social en España, determinadas variables cognitivas. Precisamente por ello, se probará si a los emprendedores sociales les condicionan las variables cognitivas que la literatura empresarial destaca como determinantes a la hora de crear una empresa (García et al., 2010) y, de ser así, si estas les influyen de manera similar tanto a los emprendedores sociales como a los comerciales.

Para conseguir dicho objetivo, el trabajo se estructura como se indica a continuación: en primer lugar, se analiza la literatura existente sobre el grupo de variables que influyen en la decisión de convertirse en emprendedor (García et al., 2010). En concreto, las variables estudiadas son la percepción de oportunidades, la sensación de que se tienen las capacidades para iniciar un negocio y el miedo ante el posible fracaso. Tras la revisión de la literatura sobre estas variables, se expone la metodología utilizada en el trabajo y, a continuación, se realiza un análisis cuantitativo sobre una muestra de 1.415 emprendedores españoles, muestra obtenida de la base de datos del GEM del año 2009. Para terminar, en un apartado final, se exponen las principales conclusiones del trabajo. 


\section{LA DECISIÓN DE CONVERTIRSE EN EMPRENDEDOR}

Los factores que influyen en la decisión de emprender no tienen por qué ser distintos en el emprendedor social (Acs et al., 2001), ya que iniciar un nuevo negocio, incluso su éxito, depende tanto de condicionantes externos e incontrolables relacionados con la cultura, educación, tecnología, normas sociales, políticas gubernamentales, etc. (Langowitz y Minniti, 2007), como de variables individuales relacionadas con la personalidad y capacidad de los emprendedores, denominadas variables cognitivas. Concretamente, este tipo de variables se encuentran relacionadas con la forma en que el individuo procesa la información del entorno, como puede ser la percepción de oportunidades, de sus propias capacidades y de los riesgos inherentes (Van Gelderen et al., 2005).

Las variables cognitivas están relacionadas con la intención de emprender y tienen carácter subjetivo, pues se fundamentan en las percepciones del individuo. La acción de emprender un negocio social, igual que cualquier otro, dependerá del reconocimiento de una oportunidad y de la formación de intenciones para actuar y responder a la oportunidad descubierta (Zander, 2004). La probabilidad de emprender aumenta cuando confluyen tres situaciones (Gnyawali y Folgel, 1994): 1) hay oportunidades, es decir, existen alternativas con expectativas de éxito para la creación de nuevas empresas; 2) el individuo está motivado y tiene la actitud requerida para crear un negocio; y 3) el individuo tiene las habilidades técnicas necesarias para iniciar y mantener una empresa. Por tanto, como cualquier iniciativa empresarial, la acción del emprendedor social se centra en dos acciones: (1) el reconocimiento de una oportunidad y (2) la formación de intenciones para responder activamente a las oportunidades descubiertas (Zander, 2004).

\section{Reconocimiento de oportunidades}

Efectivamente, un individuo se animará a emprender si detecta que hay una oportunidad de negocio que puede aprovechar (Guclu et al., 2002). Las oportunidades pueden estar delante de los individuos, algunos las verán y otros no. De los que las perciban, algunos las seguirán y otros no (Minniti y Bygrave, 
1999). Además, no suelen aparecer ante los ojos de los individuos completamente formadas, ni tampoco están allí como un tesoro perdido, esperando a ser descubiertas por la suerte o persistencia y astucia del observador, pero, solo los que las aprovechen se convertirán en emprendedores (Minniti y Bygrave, 1999).

Bajo esta perspectiva, la percepción de oportunidades es entendida como un proceso que depende de la capacidad que tengan los individuos de percibir oportunidades bajo un proceso dinámico, creativo y reflexivo. Los emprendedores cuentan con una fuerte intuición a la hora de identificar oportunidades de negocio y suelen apostar por sus ideas (Baron, 2004).

La principal diferencia entre un emprendedor comercial y un emprendedor social no es tanto el proceso de búsqueda o toma de conciencia de la existencia de una oportunidad en el mercado, sino la percepción de la oportunidad en sí misma. De hecho, lo que para un emprendedor social es una oportunidad, para un emprendedor comercial no suele ser más que un problema que otros deben resolver (Dees, 2001). Los emprendedores comerciales tienden a buscar nichos donde ganar dinero, profundizando en los ya existentes, o bien, tratando de crear nuevas necesidades en los consumidores. En cambio, las oportunidades del emprendedor social parten de problemas no resueltos en la sociedad. Más que buscar un nicho de mercado, buscan soluciones innovadoras para problemas sociales desatendidos (Austin et al., 2006).

Desde el punto de vista del emprendedor social, la oportunidad surge con la posibilidad de iniciar un negocio que tenga un impacto social positivo (Austin et al., 2006). Es ese afán por ayudar a los sectores desfavorecidos y transformar la sociedad lo que justifica la inversión de tiempo, energía y dinero que realizan (Doyle y Ho, 2010; Meyskens et al., 2010). Por consiguiente, se formula:

Hipótesis 1a: Los individuos con alta percepción de oportunidades son más propensos a ser emprendedores sociales que aquellos con baja percepción de oportunidades.

Hipótesis 1b: La percepción de oportunidades será similar entre emprendedores sociales y comerciales. 


\section{Formación de intenciones}

El proceso mediante el cual una oportunidad se convierte en una ejecución real se ve afectado por una serie de factores externos e internos. Algunas investigaciones manifiestan la importancia que tienen para emprender, no solo las capacidades y habilidades necesarias, sino también la percepción que el emprendedor tiene de ellas (Bandura, 1997; McGee et al., 2009). La autoeficacia percibida tiene sus raíces en el concepto que el individuo posee de sí mismo y refleja, por tanto, no una situación real, sino la percepción, por parte de una persona, sobre si tiene las habilidades necesarias y si es capaz de usar esas habilidades para lograr un resultado deseado (Tang, 2008).

La teoría de la autoeficacia percibida, desarrollada por Bandura (1997), ha demostrado que la probabilidad de emprender aumenta si el individuo se siente capaz. Esto es así porque sentir que uno tiene las habilidades necesarias para iniciar y dirigir una empresa facilita que el emprendedor tenga la actitud requerida para crear un negocio, incluso favorece el reconocimiento de oportunidades de negocio (Kickul et al., 2008; Rubio y Marín, 2010).

Por lo tanto, cualquier acercamiento al emprendedor social implica prestar atención, no solo a sus capacidades, sino también a la percepción que él tiene de las mismas (García et al., 2010). En este sentido, que el emprendedor social esté, en ocasiones, poseído por su idea de hacer del mundo un lugar mejor, actuando a veces en situaciones adversas de escasez de recursos e incertidumbre, hace pensar que la percepción que tiene de sus capacidades supera a la que tienen otro tipo de emprendedores. Si se analiza la conducta del emprendedor comercial se observa que, normalmente, actúa bajo un prisma racional, tratando de maximizar sus beneficios, minimizando sus costes, no dudando en poner fin a sus actividades si la falta de recursos o el mal acceso a los mismos le llevan a incurrir en pérdidas. Sin embargo, esta situación, bastante habitual en el ámbito social, no frena al emprendedor social. El individuo que persigue solucionar un problema social suele continuar con sus actividades, al pensar que es capaz de hacer más con menos, utilizando eficientemente los escasos recursos que dispone, explorando las 
opciones de captación de los mismos, desde la pura filantropía hasta los métodos comerciales (Dees, 2001).

Esta mayor autoeficacia también se ve ratificada en las investigaciones que afirman que la confianza del emprendedor sobre sus propias habilidades, conocimiento y capacidad para iniciar una empresa aumenta cuando se encuentra en estado de alerta (Langowitiz y Minniti. 2007). En las últimas décadas, la globalización y el mayor acceso a la información han hecho generar en muchos individuos dicho estado de alerta, al observar la insuficiente implicación de las instituciones públicas en los problemas sociales. Esto ha promovido el desarrollo de proyectos que tratan de acabar con la marginación o la desigualdad social, así como de pretender mejorar las condiciones de vida, trabajo o relaciones entre personas. Este argumento lleva a enunciar:

Hipótesis 2a: Los individuos con alta percepción de autoeficacia tienen más probabilidad de ser emprendedores sociales que aquellos con baja percepción de autoeficacia.

Hipótesis 2b: La percepción de autoeficacia será similar entre emprendedores sociales y comerciales.

\section{Miedo al fracaso}

Siguiendo con la formación de intenciones, otra variable que afecta al emprendedor es el riesgo que el individuo percibe y que está dispuesto a asumir. Para analizar la incertidumbre que cualquier empresa conlleva es necesario estudiar cuidadosamente los riesgos asociados. La tendencia a la asunción de riesgos se define como "la probabilidad percibida de recibir las recompensas asociadas con el éxito de una situación propuesta, que es requerido por un individuo antes de que él se sujetara a las consecuencias asociadas con el fracaso, las situaciones alternativas que establezcan una menor retribución, así como graves consecuencia que propone la situación" (Brockhaus, 1981). Si el individuo percibe que emprender es demasiado arriesgado, no se embarcará en la aventura.

El miedo al fracaso es una de las principales razones por las que la gente no elige el emprendimiento como una opción profesional, no solo por la posible pérdida económica, sino por el sentimiento de vergüenza y, posiblemente, por la exclusión 
social que provocaría el fracaso de la actividad (Ojasalo, 2004). No obstante, si el emprendedor es capaz de gestionar y controlar los riesgos, el atractivo de la iniciativa empresarial aumenta.

Esta relación, entre emprender y el miedo, ha recibido una especial atención en la literatura (Arenius y Minniti, 2005). Los resultados ponen de manifiesto que una menor percepción de posibilidad de fracasar debería aumentar la probabilidad de emprender, ya que la mayoría de individuos son adversos al riesgo y el temor al fracaso es un componente relevante del riesgo inherente a la creación de un nuevo negocio (Arenius y Minniti, 2005).

Aunque la mayoría de emprendedores opera en condiciones de riesgo, los sociales se enfrentan a un conjunto específico de desafíos, al ubicar sus empresas en zonas donde los mercados funcionan mal (Di Domenico et al., 2010). A pesar de su proximidad al riesgo, el emprendedor social no deja que el miedo le impida seguir sus visiones, asume los riesgos calculados y los gestiona a la baja, a fin de reducir el daño que resultara del fracaso (Dees, 2001). Por ello se expone:

Hipótesis 3a: Los individuos con menor percepción de miedo al fracaso son más propensos a ser emprendedores sociales que los que tienen miedo de fracasar.

Hipótesis 3b: La percepción de miedo al fracaso será similar entre emprendedores sociales y comerciales.

\section{METODOLOGÍA}

\section{Muestra y recogida de datos}

Los análisis se han realizado sobre una muestra representativa de población adulta en España. La unidad de análisis es el individuo, utilizándose parte de los datos recopilados por el proyecto GEM. Este es un proyecto multinacional que investiga la incidencia y las causas de la iniciativa empresarial, tanto dentro de un país como entre diferentes países. Los datos fueron recogidos en 2009, a través de entrevistas telefónicas con un cuestionario estandarizado. Se utilizó una muestra de adultos representativa (18-64 años), dando como resultado un total de 28.888 personas. Con el fin de asegurar que los encuestados 
reflejan correctamente la población establecida, el GEM asignó a cada entrevistado un factor de ponderación que tiene en cuenta el género y la edad. En concreto, la distribución por edad y género de las muestras se compararon con la base de datos del U.S. Census International Database del año 2002, calculándose los pesos a fin de coincidir con la muestra de esta fuente estándar de estimaciones de estructura de población. Para más información sobre el proyecto GEM y su metodología ir a Reynolds et al. (2005) y a Lepoutre et al. (2013).

\section{Medidas de las variables.}

En este apartado se explican cómo se han medido las diferentes variables objeto de estudio que influyen en la decisión del individuo a la hora de crear una empresa social.

\section{Variables dependientes}

Para identificar a los individuos involucrados en el proceso de creación de empresas se les preguntó a los encuestados si, en la actualidad, estaban tratando de poner en marcha un nuevo negocio, solos o con otras personas, o si eran trabajadores por cuenta propia. Como resultado se constituye la variable dicotómica, donde se clasifica con un 1 a aquellos individuos que contestaron positivamente, y por tanto eran emprendedores, y con un 0 a los que contestaron negativamente.

Para identificar a los individuos en el proceso de creación de una empresa social, seguidamente se les preguntó si su actividad, organización o iniciativa tenía objetivos sociales, medioambientales o si esta buscaba el bien de la comunidad. Con los resultados de esta pregunta se elaboró una nueva variable dicotómica, la cual se clasificó con un 0 a los que respondieron que no y con un 1 a los que respondieron que sí y, por tanto, eran emprendedores sociales.

\section{Variables independientes}

Las características cognitivas de los emprendedores se midieron de la siguiente manera. En primer lugar, para identificar el 
proceso de reconocimiento de oportunidad de negocio, se les preguntó a los individuos si pensaban que existían buenas oportunidades para iniciar un negocio en la zona donde vivían en los seis meses siguientes a la encuesta. Para conocer si se sienten capaces de emprender se le preguntó al individuo si creían tener el conocimiento, la habilidad y la experiencia necesaria para iniciar un nuevo negocio. Por último, para conocer si el individuo tiene miedo a fracasar se le preguntó si el miedo al fracaso sería para él un obstáculo a la hora de iniciar un nuevo negocio. Esto ha permitido crear tres variables con valor 1 cuando el encuestado respondió "sí" y con 0 cuando respondió "no".

\section{Variables control}

Tras la revisión de la literatura se han incluido tres variables de control que pueden condicionar la decisión de poner en marcha una empresa: la edad, el género y el nivel educativo (Peterman y Kennedy, 2003). Por ello, se incluyeron estas tres variables, con el fin de comprobar si las hipótesis de variables predictoras afectan al nivel de reconocimiento de la oportunidad más allá del impacto de estos controles. La edad se midió mediante una variable continua que varía desde los 18 años a los 64 , el género por una variable dicotómica donde $(0)=$ hombre y (1)=mujer y la educación por una variable de cinco categorías donde $(0)=$ ninguna formación, (1)= educación primaria, (2)= educación secundaria, (3)= educación FP superior y (4)= formación universitaria. En el análisis de regresión logística, la educación universitaria es la categoría que se utiliza como referencia, es decir, se compara si aquellos individuos con niveles de educación que no sean titulados universitarios tienen mayor o menor probabilidad de iniciar un negocio que aquellos que sí lo son.

\section{Especificación del modelo}

Formalmente, se probarán las hipótesis mediante modelos de regresión logística binomial. Esta calcula la probabilidad de que suceda un acontecimiento, en este caso es la decisión de convertirse en emprendedor o no. Con el fin de poner a prueba estas hipótesis se realizan dos modelos de regresión logística binominal. La diferencia entre los modelos radica en la muestra 
utilizada. En el modelo I se ha utilizado una muestra de 28.672 individuos, de los cuales, 1.258 son emprendedores comerciales (se han excluido los 216 emprendedores sociales). Para el Modelo II, se ha utilizado una muestra formada por 27.630 personas, de los cuales 216 son emprendedores sociales (se han excluido los 1.258 emprendedores comerciales). Asimismo, se ha utilizado la prueba de Wald para probar la significancia de los coeficientes de regresión. Con el fin de hacer más fácil la interpretación de los resultados se ha calculado el odds ratio. Este es el exponente del coeficiente de regresión. Concretamente, para las variables binarias que se aproximan a la cantidad más probable (o improbable), es el resultado (es decir, reconocimiento de la oportunidad) de estar presente entre los encuestados con un valor predictor igual a uno, en comparación con los encuestados con un valor predictor igual a cero (Hair et al., 1998).

\section{ANÁLISIS Y RESULTADOS}

La tabla 1 muestra los resultados de los dos modelos de regresión realizados. Para el modelo I, la edad se asocia negativamente con la variable dependiente, lo que indica que un emprendedor comercial es, generalmente, joven. La edad tiene una estadística de Wald de 80.953, que es significativa al nivel 0,000 . El odds ratio para esta variable es de 0,976. 
Tabla 1.

Modelo de regresión lineal

\begin{tabular}{|c|c|c|c|c|c|c|}
\hline \multirow[b]{2}{*}{ VARIABLE } & \multicolumn{3}{|c|}{$\begin{array}{c}\text { MODELO I: } \\
\text { EMPRENDEDOR COMERCIAL }\end{array}$} & \multicolumn{3}{|c|}{$\begin{array}{c}\text { MODELO II: } \\
\text { EMPRENDEDOR SOCIAL }\end{array}$} \\
\hline & B & Wald $\chi 2$ & $\operatorname{Exp}(B)$ & B & Wald $\chi 2$ & $\operatorname{Exp}(B)$ \\
\hline Edad & $-0,024^{\star \star \star}$ & 80,953 & 0,976 & $-0,003$ & 0,202 & 0,997 \\
\hline Género & $-0,302^{\star *}$ & 22,687 & 0,740 & $-0,186$ & 1,489 & 0,831 \\
\hline Nivel educativo & & 23,139 & & & 71,084 & \\
\hline Ninguno & $-0,893^{\star \star \star}$ & 15,395 & 0,409 & $-2,076^{\star \star \star}$ & 21,230 & 0,125 \\
\hline Primaria & $-0,837^{\star \star \star}$ & 18,511 & 0,433 & $-2,640^{\star \star \star}$ & 51,285 & 0,071 \\
\hline Secundaria & $-0,913^{\star \star \star}$ & 20,813 & 0,401 & $-2,176^{\star \star \star}$ & 35,001 & 0,113 \\
\hline FP Superior & $0,745^{\star \star \star}$ & 15,493 & 0,475 & $1,183^{\star \star \star}$ & 16,546 & 0,306 \\
\hline Oportunidad & $0,648^{\star \star \star}$ & 953,260 & 1,912 & $0,932^{\star \star \star}$ & 37,110 & 2,539 \\
\hline Autoeficacia & $2,466^{\star \star \star}$ & 460,827 & 11,778 & $1,181^{\star \star \star}$ & 36,322 & 3,259 \\
\hline Miedo al fracaso & $-0,650^{\star \star \star}$ & 100,935 & 0,522 & 0,196 & 1,677 & 0,822 \\
\hline \multicolumn{7}{|c|}{ Modelo de diagnóstico } \\
\hline Muestra & \multicolumn{3}{|c|}{28.672} & \multicolumn{3}{|c|}{27.630} \\
\hline Modelo $\chi 2$ & \multicolumn{3}{|c|}{$18,210^{\star \star \star}$} & \multicolumn{3}{|c|}{$19,019^{\star \star}$} \\
\hline $\begin{array}{l}\% \text { predicciones } \\
\text { correctas }\end{array}$ & \multicolumn{3}{|c|}{94,8} & \multicolumn{3}{|c|}{99,2} \\
\hline $\mathrm{R}^{2}$ & \multicolumn{3}{|c|}{0,174} & \multicolumn{3}{|c|}{0,094} \\
\hline
\end{tabular}

$\left({ }^{* *}\right): p<0,01 ;\left(^{* *}\right): p<0,05 ;\left({ }^{*}\right): p<0,1$.

Fuente: Elaboración propia.

El género también se asocia negativamente con la variable dependiente, lo que apunta a que el emprendedor comercial es, generalmente, un hombre. El género tiene una estadística de Wald igual a 22,687, que es significativa al nivel 0,000. El odds ratio para esta variable es 0,740 .

Por último, el nivel educativo ofrece resultados negativos en su relación con la variable dependiente, a excepción del nivel FP superior, lo que sugiere que un emprendedor comercial es, generalmente, una persona con un nivel de estudios elevado. El nivel de educación tiene una estadística de Wald igual a 15.395 para ninguna educación, 18.511 para educación básica, 20.813 para 
educación secundaria y 15.493 para FP superior. Todos ellos son significativos al nivel de 0,000.

De acuerdo con la literatura existente, los resultados indican que la edad, género y nivel formativo condicionan al emprendedor. Por otra parte, todas las variables de percepción introducidas son altamente significativas. En particular, los resultados muestran que la autoeficacia y la percepción de oportunidades son positivas y están significativamente relacionadas con ser emprendedor comercial.

Percibir oportunidades ofrece una estadística de Wald de 95,260 , que es significativa al nivel 0,000. El odds ratio para esta variable es 1,912. Esto apunta hacia que las personas que perciben oportunidades tienen casi 1,9 veces más probabilidad de ser emprendedores comerciales que los que no perciben oportunidades.

Como se ha comentado, la autoeficacia es positiva y está significativamente relacionada con ser emprendedor comercial. Esta variable tiene una estadística de Wald igual a 460.827, que es significativa en el nivel 0,000 . El odds ratio para la confianza en la variable de habilidades es 11,778. Esto indica que aquellos que se perciben capaces tienen casi 12 veces más probabilidad de ser emprendedores comerciales que los que no creen tener los conocimientos necesarios.

El miedo al fracaso se asocia negativamente con la variable dependiente, lo que apunta que un emprendedor comercial es una persona con baja percepción de miedo. Esta variable tiene una estadística de Wald igual a 100,935 , que es significativa al nivel 0,000 . El odds ratio para esta variable es 0,522 . Esto sugiere que las personas con miedo a fracasar tienen solo la mitad de probabilidad de iniciar un nuevo negocio que aquellos que no tienen miedo.

Por tanto, es posible definir el perfil del emprendedor comercial como un hombre joven, con elevado nivel educativo, alta percepción de oportunidades y de autoeficacia, así como bajo nivel de miedo.

Por su parte, en el modelo II se observan los resultados para la muestra de los emprendedores sociales. En primer lugar, se debe destacar que, a diferencia de lo observado con el emprendedor comercial, en este modelo no todas las variables han 
obtenido una relación significativa con el hecho de convertirse en emprendedor social. En concreto, se observa que no existe ninguna relación significativa entre la variable dependiente y dos de las variables control, edad y género, y la variable independiente miedo al fracaso. Por tanto, se puede decir que convertirse en emprendedor social en España no depende de la edad del individuo, ni del género ni de su miedo ante el posible fracaso.

Para el emprendedor social, al igual que para el comercial, el nivel educativo se asocia negativamente con la variable dependiente en todos los niveles excepto en el de FP superior. Este hecho indica que los emprendedores sociales suelen tener un nivel educativo elevado. La variable nivel de educación tiene una estadística de Wald igual a 21,230 para ninguna educación, 51,285 para educación básica, 35,001 para educación secundaria y 16,546 para FP superior. Todos ellos son significativos en el nivel de 0,000.

Además, las variables autoeficacia y percepción de oportunidades de negocio han obtenido resultados positivos y se encuentran significativamente relacionadas con ser un emprendedor social, hecho que también se ha observado para el caso del emprendedor comercial.

La variable percepción de oportunidades tiene una estadística de Wald equivalente a 37,110 , lo cual es significativo en el nivel de 0,000 . El odds ratio para esta variable es 2,539. Esto indica que aquellos que perciben oportunidades tienen casi 2,5 veces más probabilidad de ser emprendedores sociales que los que no perciben oportunidades, dato superior al obtenido por el emprendedor comercial $(1,912)$.

La autoeficacia es positiva y está significativamente relacionada con ser un emprendedor social. La variable tiene una estadística de Wald igual a 36,322, lo cual es significativo en el nivel 0,000 . El odds ratio para la confianza en la variable habilidades es 3,259 . Esto sugiere que aquellos que se perciben capaces tienen casi 3,259 veces más probabilidad de ser emprendedores sociales que los que no creen tener los conocimientos y habilidades necesarias. Se debe destacar que, a pesar de haber obtenido un odds ratio elevado, este es muy inferior al observado en el modelo del emprendedor comercial $(11,778)$. 
Por lo tanto, mientras que el emprendedor comercial es un hombre, joven, con elevado nivel educativo, que percibe oportunidades, que se siente capaz y que tiene bajos niveles de miedo al fracaso, el emprendedor social es una persona con elevado nivel educativo, con alta percepción de oportunidades y de autoeficacia percibida.

En cuanto a las hipótesis planteadas en este trabajo, se ha comprobado que la 1a y 2 a pueden ser aceptadas, en cambio las hipótesis $1 \mathrm{~b}$ y $2 \mathrm{~b}$ deben ser aceptadas parcialmente. Es cierto que la relación de la variable percepción de oportunidades (hipótesis $1 b$ ) y autoeficacia (hipótesis $2 b$ ) con el hecho de convertirse en emprendedor social o comercial es similar, pero si se atiende a sus odds ratios se observa que las probabilidades difieren, siendo la primera de ellas mayor para el caso social (2,5 frente al 1,9 del emprendedor comercial), mientras que la segunda es bastante menor (3,259 frente al 11,778 del emprendedor comercial), de ahí que ambas hipótesis solo puedan ser aceptadas parcialmente. Por último, los resultados indican que tanto la hipótesis $3 a$ como la $3 b$ debe ser rechazadas, al encontrarse que, a diferencia del emprendedor comercial, el social no se encuentra influido por esta variable.

\section{DISCUSIÓN Y CONCLUSIONES}

La contribución principal de esta investigación es la visión que se ofrece de la figura del emprendedor social. En primer lugar, este estudio contribuye a la literatura empresarial social, tras testar el modelo del emprendedor comercial en una muestra de emprendedores social y poner de manifiesto que existen diferencias entre ambos tipos de emprendedores, a través de un marco analítico sobre cómo abordar el proceso emprendedor social de manera sistemática y eficaz. En concreto, los resultados muestran que, mientras que los emprendedores comerciales, por lo general, son varones jóvenes, con elevado nivel educativo, alta autoeficacia, alta percepción de oportunidad y bajo nivel de miedo al fracaso, la probabilidad de convertirse en un emprendedor social no depende de variables como el género, la edad y el miedo al fracaso. 
El término emprendimiento social puede ser nuevo, pero el fenómeno en sí no lo es (Dees, 2001). Siempre ha habido personas que inician nuevos proyectos empresariales con los que se proponen la mejora social. Sin embargo, el auge reciente del emprendimiento social abre algunas líneas de investigación para el desarrollo de este fenómeno empresarial, presentando un análisis exploratorio comparativo de los elementos aplicados al emprendimiento comercial, fenómeno que más extensamente ha sido estudiado, siendo dichos elementos transferibles a la figura del emprendedor social (Austin et al., 2006).

De ahí, que el propósito de esta investigación fuera explorar el grado en que los elementos aplicables al emprendimiento comercial, profundamente analizado, son transferibles al caso social. Con la investigación cuantitativa se ha probado el modelo de emprendedor comercial en la muestra de emprendedores sociales. De los hallazgos destaca la existencia de diferencias entre ambos emprendedores.

En primer lugar, los resultados sugieren que la probabilidad de convertirse en emprendedor social no tiene relación con el género, mientras que para el emprendimiento comercial los resultados muestran que es más probable que el hombre cree empresas. Las mujeres han hecho grandes avances en los últimos años, al cerrar la brecha de la iniciativa empresarial. Sin embargo, persiste la preocupación de que las mujeres están subrepresentadas entre los emprendedores. Como apunta la literatura, las mujeres no tienen las mismas motivaciones que los hombres (Brush, 1992; Eddleston y Powell, 2008; Mueller y Conway DatoOn, 2008; Díaz y Jiménez, 2010). De ahí que se afirme que los hombres se identifican más con objetivos económicos, mientras que las mujeres son más propensas a buscar altruistamente el bien común (Themudo, 2009). Por ello, la literatura empresarial social sitúa a la mujer como objetivo clave del desarrollo de este fenómeno empresarial (Hechavarría et al., 2010), aunque hay que puntualizar que, en esta investigación, no se ha podido demostrar tal afirmación.

En segundo lugar, la probabilidad de ser emprendedor comercial es más alta entre los jóvenes. Este hecho coincide con la literatura existente, para la cual la creación de nuevas empresas parece ser un juego de hombres jóvenes (García et al., 2010; 
Lévesque y Minniti, 2006; Arenius y Minniti, 2005; Reynolds et al., 2005). La relación entre la edad y la probabilidad de emprender alcanza su punto máximo a una edad relativamente temprana y disminuye a partir de entonces (Lévesque y Minniti, 2006). Sin embargo, los resultados de los emprendedores sociales son muy diferentes. Para ellos, no existe una relación significativa entre la decisión de convertirse en emprendedor social y la edad. Así que, en este caso, la probabilidad de iniciar una nueva empresa para hacer frente a necesidades sociales no depende de la edad del emprendedor.

Los resultados muestran una relación positiva entre el nivel formativo y la probabilidad de emprender comercial o socialmente. Resulta llamativo que, tanto para los emprendedores comerciales como para los sociales, se observa que las personas que tienen un título de FP superior son más propensas a iniciar una empresa, frente a aquellas que tienen un título de educación más bajo o, incluso, universitario.

Así pues, se puede concluir que los emprendedores comerciales son hombres jóvenes, a pesar de que ser un emprendedor social no depende del género o edad. Además, para ambos tipos de emprendimiento se han encontrado emprendedores con elevado nivel educativo. Los resultados del estudio también muestran que existen diferencias entre los emprendedores cuando se hablan de las variables independientes: (1) percepción de oportunidades, (2) autoeficacia percibida y (3) miedo al fracaso.

En concreto, los resultados indican que la percepción de oportunidades y de autoeficacia percibida se asocia positivamente con la decisión de convertirse en emprendedor. La autoeficacia emerge como el componente más importante de la decisión de iniciar un nuevo negocio, tanto comercial como social, como muestran los estudios previos (Arenius y Minniti, 2005; Baron, 2004), seguido por la percepción de oportunidad (Kirzner, 1973). El impacto positivo de estas variables es difícil de conciliar con la anterior teoría de iniciativa empresarial, según la cual la confianza en sus habilidades y la percepción de oportunidades no explotadas son una condición necesaria para la acción empresarial (García et al., 2010; Arenius y Minniti, 2005; Kirzner, 1973). Mientras que autores como Jenssen y Havnes (2002) afirman que la persona que decide iniciar un negocio debe tener una inclinación innata, otros como Kruegel 
y Brazeal (1994) indican que los emprendedores no nacen con la capacidad necesaria, sino que necesitan ser formados para ello. Además, como se ha puesto de manifiesto, se ha encontrado una relación positiva entre la percepción de oportunidades y la probabilidad de convertirse en emprendedor.

El miedo se asocia negativamente con la decisión de emprender comercialmente, resultados coincidentes con estudios previos. Como Arenius y Minniti (2005) sugieren, la percepción de riesgo de un individuo reduce los incentivos empresariales al aumentar la probabilidad percibida de fracaso. Sin embargo, para el caso social no se han obtenido resultados significativos, por lo que, el miedo a fracasar no reduce la probabilidad de emprender socialmente. Este resultado se puede explicar con la teoría del intercambio social. Esta parte de la idea de que los participantes del intercambio relacional pueden esperar obtener satisfacciones personales no económicas (Dwyer et al., 1987). Las transacciones de empresas comerciales suelen considerarse como un intercambio monetario por un producto o servicio, mientras que en el caso social no todos los beneficios son de carácter económico. De hecho, el principal beneficio de este tipo de emprendimiento no tiene valor material, de ahí que no sea posible ponerle precio exacto a todo, como por ejemplo la aprobación social y el respeto (Blau, 1968) y, además, a menudo las recompensas sociales se valoran más que las económicas.

Los resultados de este trabajo tienen implicaciones potencialmente importantes. Por un lado, este estudio sobre la naturaleza de la iniciativa empresarial social contribuye a la creciente literatura que cuestiona las diferencias entre el emprendedor social y comercial. Por otra parte, los resultados refuerzan la importancia del emprendedor social, que debe estar en el centro de diversas cuestiones políticas, ya que, como dijo Dees (2001), son necesarios emprendedores sociales que ayuden a encontrar nuevos caminos, si se quiere conseguir la mejora social.

Para los investigadores existen multitud de ricas líneas para futuros estudios. El análisis comparativo revela puntos comunes entre los emprendedores sociales y comerciales, pero también ponen de manifiesto algunas diferencias importantes respecto a la misión, capacidades, recursos y medidas de desempeño, entre otras. Para avanzar en el conocimiento del emprendedor social 
sería interesante profundizar en la variable género, así como en la persistencia, las relaciones sociales, la creatividad y el liderazgo, entre otras, variables que la literatura identifica como relevantes y que, por tanto, también podrían ser consideradas en futuras investigaciones.

En conclusión, este trabajo contribuye a la literatura empresarial mediante el análisis del emprendimiento social a través de un estudio empírico. Es nuestra esperanza que esta investigación promueva la comprensión del emprendimiento social, así como que se estimule y facilite la labor de exploración científica de las cuestiones que rodean, excepcionalmente, este fenómeno empresarial.

\section{REFERENCIAS BIBLIOGRÁFICAS}

Acs, Z. Bosma, N. y Sternberg, R. (2011). The dynamics of entrepreneurship: Theory and evidence, chapter Entrepreneurship in World Cities. OUP: Oxford University Press.

Acs, Z. y Audretsch, D. (2001). The emergence of the entrepreneurial society. Stokholm Sweden: Swedish Fundation for Small Business Review.

Arenius, P. y Minniti, M. (2005). Perceptual variables and nascent entrepreneurship. Small Business Economic, 24, 223-247.

Ashoka España (2018). Portal web Ashoka España, disponible en: http://spain.ashoka.org/

Austin, J., Stevenson, H. y Wei-Skillern, J. (2006). Social and Comercial entrepreneurship: same, different, or Both?. Entrepreneurship Theory and Practice, 30(1), 1-22.

Bandura, A. (1997). La auto-eficacia: El ejercicio del control. Nueva York: WH Freeman.

Baron, R. (2004). The Cognitive Perspective: A Valuable Tool of Answering Entrepreneurship's Basic "Why" Questions. Journal of Business Venturing, 19, 221-240.

Blau, P.M. (1964). Exchange and Power in Social Life. New York: John Wiley \& Sons.

Bornstein, D. y Davis, S. (2010). Social Entrepreneurship. What everyone needs to know. Oxford: Oxford University Press. 
Brockaus, R.H. (1981). Risk taking propensity of entrepreneurs. Academy of Management Journal, 23, 509-520.

Brush, C. (1992). Research on women business owners: past trends, a new perspective and future directions. Entrepreneurship Theory and Practice, 16(4), 5-30.

Dees, G. (1998). The Meaning of Social Entrepreneurship, The Kaufmann Center for Entrepreneurial Leadership and Ewing Marion Kaufmann Foundation, disponible en: http://www.redalmarza.cl/ing/pdf/TheMeaningofsocialEntrepreneurship.pdf

Dees, J. G. (2001). The Meaning of social entrepreneurship, disponible en: http://www.fuqua.duke.edu/centers/case/documents/dees SE.pdf

De la Vega, I., Coduras, A., Cruz, C., Justo, R. y González, I. (2010). Informe GEM España 2009. Madrid: Instituto de Empresa.

Díaz, Ma . C. y Jiménez, J.J. (2010). Recursos y resultados de las pequeñas empresas: nuevas perspectivas del efecto género. Cuadernos de Economía y Dirección de la empresa, 42(marzo), 151-176.

Di Domenico, M. L., Haugh, H. y Tracy, P. (2010). Social Bricolage: Theorizing social value creation in social enterprises. Entrepreneurship Theory and Practice, 34(4), 681-703.

Doyle, P. y Ho, M. (2010). How Opportunities Develop in Social Entrepreneurship. Entrepreneurship Theory and Practice, Special Issue: Social Entrepreneurship, 34(4), 635-659.

Dwyer, F. R., Schurr, P.H. y Sejo, O.H. (1987). Developing BuyerSeller Relationships. Journal of Marketing, 51(April), 11-27.

Eddleston, K.A. y Powell, G.N. (2008). The role of gender identity in explaining sex differences in business owners' career satisfier preferences. Journal of Business Venturing, 23(2), 244-256.

García, C., Martínez, A. y Fernández, R. (2010). Características del emprendedor influyentes en el proceso de creación empresarial y en el éxito esperado. Revista Europea de Dirección y Economía de la Empresa, 19(2), 31-48. 
Gnyawali, D.R. y Fogel, D.S. (1994). Environments for entrepreneurship development: key dimensions and research implications. Entrepreneurship: Theory and Practice, Summer, 43-62.

Guclu, A.J., Dees, J.G. y Anderson, B.B. (2002). The process of social entrepreneurship: creating opportunities worthy of serius pursuit. Center for the advancement of social entrepreneurship, 1-15.

Hair, J., Anderson, R., Tatham, R., Black. W. (1998). Multivariate data analysis. 5th ed. New York: Prentice Hall College Div.

Hechavarría, D. M., Ingram, A., Justo, R. y Terjesen, S. (2012). Are Women More Like to Pursue Social and Environmental Entrepreneurship?. En K. D. Hughes y J. E. Jennings (Ed.). Global Women's Entrepreneurship Research: Diverse Settings, Questionsand Approaches (pp. 135-147). Edward Elgar Publishing.

Jenssen, J.I. y Havnes, P.A. (2002). Public intervention in the entrepreneurial process. A study based on three Norwegian cases. International Journal of Entrepreneurial Behavior and Research, 8(3), 173-187.

Kickul, J., Wilson, F., Marlino, D. y Barbosa, S.D. (2008). Are Misalignments of perceptions and self-efficacy causing gender gaps in entrepreneurial intentions among our nations' teens?. Journal of Small Business and Enterprise Development, 15(2), 321-335.

Kirzner, I. (1973). Competition and entrepreneurship. Chicago: The University of Chicago Press.

Kristuck, G. M. y Beamish, P. W. (2010). The interplay of form, structure, and embeddedness in social intrapreneurship. Entrepreneurship Theory and Practice, 34(04), 735-761.

Krueger, N.F. y Brazeal, D.V. (1994). Entrepreneurial potential and potential entrepreneurs. Entrepreneurship Theory and Practice, 18(1), 91-104.

Langowitz, N. y Minniti, M. (2007). The Entrepreneurial Propensity of Women, Entrepreneurship Theory and Practice, 31(3), 341-364.

Lepoutre, J., Justo, R., Terjensen, S. y Bosma, N. (2013). Designing a global standardized methodology for measuring social entrepreneurship activity: the Global Entrepreneurship Monitor social entrepreneurship study. Small Business Economics, 40(3), 693-714. 
Lévesque, M. y Minniti, M. (2006). The effect of aging on entrepreneurial behavior. Journal of Business Venturing, 21, 177194.

McGee, J.E., Peterson, M., Mueller, S.L. y Sequeira, J.M. (2009). Entrepreneurial selfefficacy: Refining the measure. Entrepreneurship Theory and Practice, 33(4), 965-988.

Meyskens, M., Robb-Post, C., Stamp, J.A., Carsrud, A.L. y Reynolds, P.D. (2010). Social ventures from a resource-based perspective: an exploratory study assessing global Ashoka fellows. Entrepreneurship Theory and Practice, 34(4), 661-680.

Minniti, M. y Bygrave, W. (1999). The microfoundations of entrepreneurship. Entrepreneurship Theory and Practice, 23(4), 41-52.

Mueller, S. y Conway Dato-on, M. (2008). Gender-role orientation as a determinant of entrepreneurial self- efficacy. Journal of Developmental Entrepreneurship, 13(1), 3-20.

Muñoz Serrulla, M. T. (2004). Francisco Piquer y la creación del Monte de Piedad de Madrid. (1702-1739): Moneda espiritualizada y su proyección en Indias. Tesis Doctoral Universidad Complutense de Madrid, disponible en: http://eprints.ucm. es/tesis/ghi/ucm-t28042.pdf

Nicolás Martínez, C. (2014). Emprendimiento Social. Tesis Doctoral Universidad de Murcia, disponible en: https://digitum.um.es/jspui/bitstream/10201/39915/1/Catalina\%20 Nicol\%C3\%A1s\%20Mart\%C3\%ADnez.pdf

Nicolás, C., Rubio, A. y Fernández-Laviada, A. (2018). Cognitive Determinants of Social Entrepreneurship: Variations According to the Degree of Economic Development, Journal of Social Entrepreneurship, 9(2), 154-168.

Ojasalo, J. (2004). Key Network Management. Industrial Marketing Management, 33, 195-204.

Peredo, A.M. y Chrisman, J.J. (2006). Toward a Theory of Community-Based Enterprise. Academy of Management Review, 31(2), 309-328.

Peterman, N. E. y Kennedy, J. (2003). Enterprise education: Influencing students' perceptions of entrepreneurship. Entrepreneurship theory and practice, 28(2), 129-144. 
Reynolds, P.D., Bosma, N., Autio, E., Hunt, S., De Bono, N., Servais, I., López-García, P. y Chin, N. (2005). Global Entrepreneurship Monitor: data collection design and implementation 1998-2003". Small Business Economic, 24(3), 443-456.

Rubio, A. y Marín, L. (2010). Social and commercial entrepreneurship: a comparative analysis, Paper presentado a 7Th Annual Satter Conferences on Social Entrepreneurship, Nueva York 3-5 de Noviembre, 1-30.

Short, J. C., Moss, T. W. y Lumpkin, G. T. (2009). Research in social entrepreneurship: past contributions and future opportunities. Strategic Entrepreneurship Journal, 3(2), 161-194.

Seelos, C. y Mair, J. (2007). Profitable Business Models and Market Creation in the Context of Deep Poverty: A Strategic View. Academy of Management Perspectives, 21(4), 49-63.

Tang, J. (2008). Ambiental generosidad para los empresarios: el estado de alerta Empresarial y el compromiso. Revista Internacional de Conducta Empresarial y de Investigación, 14(3), 128-151.

Themudo, N.S. (2009). "Gender and the Nonprofit sector". Nonprofit and Voluntary Sector Quarterly, 38(4), 663-683.

Van Gelderen, M., Thurik, R. y Bosma, N. (2005). Success and Risk Factors in the Pre-Startup Phase. Small Business Economics, 24(4), 365-380.

Zahra, S.A., Gedajlovic, E., Neubaum, D.O. y Shulman, J.M. (2009). A typology of social entrepreneurs: Motives, search processes and ethical challenges. Journal of Business Venturing, 24, 519-532.

Zander, I. (2004). El espíritu emprendedor en el ámbito geográfico. Fundamentos conceptuales e implicaciones para la formación de nuevos clúster. Cuadernos de Economía y Dirección de la Empresa, 20, 9-34. 